\title{
ISG20L2 as a Novel Prognostic Biomarker Facilitates the Progression of Pancreatic Cancer Via Glycolysis
}

\section{Jianming Wei}

Tianjin Medical University General Hospital https://orcid.org/0000-0002-1574-2109

\section{Xibo Gao}

Tianjin Children's Hospital

\section{Bingbing Ren}

Tianjin Medical University General Hospital

\section{Daqing Sun}

Tianjin Medical University General Hospital

\section{Tong Liu(D714611908@qq.com )}

Tianjin Medical University General Hospital https://orcid.org/0000-0003-2380-8216

\section{Primary research}

Keywords: ISG20L2, pancreatic cancer, glycolysis, invasion, prognosis

Posted Date: October 14th, 2021

DOI: https://doi.org/10.21203/rs.3.rs-900506/v2

License: (c) (i) This work is licensed under a Creative Commons Attribution 4.0 International License. Read Full License 


\section{ISG20L2 as a novel prognostic biomarker facilitates the progression of} pancreatic cancer via glycolysis

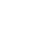
Jianming Wei ${ }^{1^{*}}$, Xibo Gao ${ }^{2 *}$, Bingbing Ren ${ }^{3 *}$, Daqing Sun ${ }^{4 \#}$, Tong Liu ${ }^{5 \#}$

1,5 Department of General Surgery, Tianjin Medical University General Hospital, Tianjin, 300052, China.

2 Department of Dermatology, Tianjin Children's Hospital, Tianjin, 300074, China.

3,4 Department of Paediatric Surgery, Tianjin Medical University General Hospital, Tianjin, 300052, China.

* These authors contributed equally to the work.

\# Co-Corresponding authors: Tong Liu, Department of General Surgery, Tianjin Medical University General Hospital, Tianjin, 300052, China. Email: 714611908@qq.com. Daqing Sun, Department of Paediatric Surgery, Tianjin Medical University General Hospital, Tianjin, 300052, China. Email: sdqchris2019@tmu.edu.cn.

\section{Abstract}

Background: Longstanding type 2 diabetes mellitus (T2DM) is an increased risk of pancreatic cancer (PC) in western populations, and PC is also a cause of T2DM. However, the association of glucose metabolism between T2DM and PC remains unclear.

Methods: Differentially expressed genes (DEGs) were identified by bioinformatic analysis from Gene Expression Omnibus (GEO) datasets GSE20966 and GSE16515, respectively. Gene Ontology (GO), Kyoto Encyclopedia of Genes and Genomes (KEGG) pathway, Gene Set Enrichment Analysis (GSEA), the Kaplan-Meier (KM) Plotter and Tumor Immune Estimation Resource (TIMER) database were applied. Pancreatic cancer cell lines and primary PDAC samples were used. Cell culture, 
immunohistochemistry (IHC), siRNA transfection, Western blot, RT-PCR, and migration assay, animal xenograft model studies and statistical analysis were performed in this study.

\section{Results:}

We identified 64 DEGs in GSE20966 of T2DM, and 296 DEGs were identified in GSE16515 of pancreatic cancer, respectively. T2DM-DEGs were mainly enriched in synaptic vesicle cycle, protein export. KEGG pathways in pancreatic cancer included spliceosome, RNA transport. Here, ISG20L2 was identified as only a co-expressed gene between T2DM and PDAC. We found that the expression of ISG20L2 was associated with tumor immune cell infiltration. ISG20L2 was significantly upregulated in PDAC and associated with prognosis of PDAC patients. Moreover, ISG20L2 expression was regulated by GLUT1, HK2, LDHA, PKM1 and PKM2 related with glycolysis in PDAC. ISG20L2 promoted PDAC cell proliferation and migration both in vitro and in vivo.

Conclusion: This study showed that ISG20L2 promoted the progression and ISG20L2 may be a potential therapeutic strategy in PDAC.

Keywords: ISG20L2, pancreatic cancer, glycolysis, invasion, prognosis

\section{Introduction}

Pancreatic cancer (PC) is the seventh leading cause of cancer death in the world, one of the most fatal malignancies ${ }^{1}$. While pancreatic cancer therapy may be differentiated, the overall 5-year survival of pancreatic cancer is less than $5 \%^{2}$ and remains one of the lowest in all cancers. Increasing evidence suggested that type 2 diabetes mellitus (T2DM) was a risk factor for pancreatic cancer. Previous study suggested that T2DM is associated with hyperglycemia and a risk to develop pancreatic ductal adenocarcinoma (PDAC) ${ }^{3}$. The relationship between T2DM and pancreatic cancer is complex. This causal relationship between T2DM and PDAC remains unclear.

Glucose metabolism is a critical element in T2DM and PDAC. Skytte, M. J. et al. reported that carbohydrate restriction affected glucose metabolism in T2DM ${ }^{4}$. Mason, I. C. et al. found that glucose metabolism in T2DM was regulated by the circadian system and impaired insulin sensitivity ${ }^{5}$. Interestingly, glucose metabolism is also 
associated with cancer growth and progression, especially in PDAC ${ }^{6}$. In this study, we firstly identified differentially expressed genes (DEGs) of T2DM and PC. Secondly, we elucidated molecular functions of T2DM-related DEGs (T2DMDEGs) and pancreatic cancer-related DEGs (PC-DEGs). Thirdly, ISG20L2 was identified a co-expressed gene and we performed a bioinformatic analysis of ISG20L2. Finally, ISG20L2 was a prognostic biomarker promoting proliferation and migration in PDAC associated with glycolysis. Taken together, ISG20L2 may be a potential mechanism and therapeutic target of pancreatic cancer.

\section{Results}

Development and identification of differentially expressed genes in T2DM and PDAC

The Study flowchart was shown in Fig.1.

Selecting GEO database mRNA expression array, a total of 64 DEGs were identified including 33 down-regulated genes and 31 up-regulated genes in GSE20966 (Fig.2A,2B). Beta-cells were acquired from pancreatic tissue sections using the laser capture microdissection technique ${ }^{7}$. We next analyzed DEGs in PDAC. In pancreatic cancer data GSE16515, a total of 296 DEGs were screened out with 71 genes overexpression and 225 genes low expression (Fig.2C, 2D).

\section{Functional enrichment analysis}

Gene Ontology (GO) and Kyoto Encyclopedia of Genes and Genomes (KEGG) pathway analysis were performed to assess the molecular functions of DEGs. GO terms included biological processes (BP), cellular components (CC) and molecular functions (MF). As shown in Fig.2E, the top 3 GO terms of DEGs in T2DM were microtubule depolymerization, terminal bouton, neuropeptide Y receptor activity. In GO terms of DEGs in PDAC, the top 3 terms were ribonucleoprotein complex localization, chromosomal region and structural constituent of nuclear pore (Fig.2F). Further investigation showed that T2DM-DEGs were mainly enriched in synaptic vesicle cycle, protein export, collecting duct acid secretion, SNARE interactions in vesicular transport, vibrio cholerae infection (Fig.2G). However, KEGG pathway analysis in pancreatic 
cancer included spliceosome, RNA transport, homologous recombination, proteasome, fanconi anemia pathway (Fig.2H).

\section{Identification of $I S G 20 L 2$ as a prognostic biomarker}

In the present study, ISG20L2 was identified only one co-expressed DEG in GSE20966 and GSE16515 datasets using online database VENNY 2.1 (Fig.3A). To explore the regulatory role of ISG20L2, we firstly analyzed its expression. ISG20L2 was significantly overexpressed in T2DM and PDAC (Fig.3B, 3C). Further exploring revealed that the expression of ISG20L2 was overexpressed in PDAC from TCGA database (Fig.3D). The Human Protein Atlas (HPA) (http://www.proteinatlas. org/) ${ }^{8}$ was applied to explore ISG20L2 expression in PDAC tissues (Fig.3E). To explore the relationship between the expression of ISG20L2 and overall survival, we found that high expression of ISG20L2 associated with poor overall survival (Fig.3F).

\section{ISG20L2 is overexpressed in PDAC as an independent prognostic factor}

Besides, we found that the ISG20L2 mRNA level in PDAC cells was also significantly increased compared to HPDE6-C7 (Fig.3G). Moreover, ISG20L2 was notably increased in PDAC patient tissues relative to the adjacent normal tissue (Fig. $3 \mathrm{H}$ ). To validate the relationship of the expression of ISG20L2 with clinicopathological characteristics, we performed IHC staining for ISG20L2 in PDAC tissue microarray, described previously 9. In the present study, we showed that the expression of ISG20L2 was significantly associated with overall survival $(\mathrm{P}=0.0030)$, stage $(\mathrm{P}=0.0030)$ and vascular invasion $(\mathrm{P}=0.0255)$ (Fig.3I-3L). In the univariate Cox regression analysis, we found that age $(\mathrm{HR}=1.03,95 \% \quad \mathrm{CI}=1.01-1.05, \mathrm{P}=0.017)$, stage $\quad(\mathrm{HR}=1.46,95 \% \quad \mathrm{CI}=1.07-1.99$, $\mathrm{P}=0.017), \quad I S G 20 L 2$ expression $(\mathrm{HR}=1.64,95 \% \mathrm{CI}=1.22-2.20, \mathrm{P}=0.0010)$ were significantly correlated with overall survival. Furthermore, in the multivariate Cox regression analysis, age $(\mathrm{HR}=1.04,95 \% \mathrm{CI}=1.01-1.07, \mathrm{P}=0.0036)$ and $\mathrm{ISG} 20 \mathrm{~L} 2$ expression $(\mathrm{HR}=1.75,95 \% \mathrm{CI}=1.30-2.36, \mathrm{P}=0.00029)$ were found to be an independent prognostic factor for patients with pancreatic cancer. As shown in Table 1.

Gene set enrichment analysis of ISG20L2 and correlation with immune cell infiltration in PDAC 
To explore the potential function of ISG20L2 in PDAC, GSEA software was performed to find KEGG pathways enriched in the 89 highly-expressed samples. ISG20L2 mainly enriched in "Rig-I-like receptor signaling pathway", "spliceosome", "aminoacyl tRNA biosynthesis", "ubiquitin mediated proteolysis", "erbb signaling pathway" and "pancreatic cancer" in this study $(\mathrm{P}<0.05)$ (Fig.4A-4G). The above results demonstrated that ISG20L2 exerted the relationship with metabolism in pancreatic cancer.

To evaluate the relationship between immune cell infiltration and ISG20L2 expression, the TIMER database was used in this study. The result demonstrated that there was a positive correlation between ISG2OL2 expression and the infiltration of CD4+ T cells $\left(\right.$ Cor $=0.014, p=7.83 \mathrm{e}^{-01}$; Fig.4I). Dendritic cells $\left(\right.$ Cor $=-0.083, p=1.09 \mathrm{e}^{-01}$; Fig.4L), neutrophils (Cor $=-0.072, p=7.83 \mathrm{e}-01 ;$ Fig.4K), CD8+ T cells $(\mathrm{Cor}=0.134$, $\mathrm{p}=1.01 \mathrm{e}-02 ;$ Fig.4H), B cell $($ Cor $=-0.034, \mathrm{p}=5.11 \mathrm{e}-01 ;$ Fig.4M), macrophages $($ Cor $=-0.125, \mathrm{p}=1.6 \mathrm{e}-02 ;$ Fig.4J) were negatively associated with ISG20L2 expression.

\section{Correlation of ISG20L2 with glycolysis}

To verify the role of $I S G 20 L 2$ in glucose metabolism, especially in glycolysis, we firstly used GEPIA database and explored the relationship between the expression of ISG2OL2 and GLUT1, HK2, LDHA, PKM which were the key enzyme in glycolysis. Interestingly, we found that the expression of ISG2OL2 was significantly associated with the expression of GLUT1, HK2, LDHA, PKM (Fig.5A-5D). Western blot analysis of transfection of si-NC and si- ISG20L2 in SW1990 cells showed that ISG20L2 significantly promoted the expression of GLUT1, HK2, LDHA, PKM1 and PKM2 in vitro (Fig.5E,5F). The result was also validated in vivo (Fig.5G,5H).

\section{ISG20L2 promotes PDAC cell proliferation and metastasis in vitro and vivo}

To investigate the biological effect of ISG20L2 in PDAC, we performed explored the ability of ISG20L2 on migration and invasion in PDAC cells. Transwell migration and Matrigel invasion assays showed that ISG20L2 downregulation and upregulation significantly inhibited and improved the migratory and invasive capabilities of PDAC cells, respectively (Fig. 6A, 6C). This study indicated that PDAC cells proliferation was 
significantly inhibited in si-ISG20L2 compared to si-NC cells (Fig. 6B). Collectively, these results showed that $I S G 20 L 2$ facilitated the proliferation, migration, and invasion of PDAC cells.

To elucidate the oncogenic role of ISG20L2 in PDAC in vivo, we performed subcutaneous tumorigenesis experiment using si-NC and si-ISG20L2 SW1990 cells. The data suggested that the ISG20L2 knockdown effectively reduced compared to siNC group in tumor weight and volume (Fig.6D-6F). Next, qPCR analysis was performed to confirm ISG20L2 expression in xenografted tumor tissues. The result revealed that ISG20L2 was overexpressed significantly in si-NC than si-ISG2OL2 (Fig.6G). IHC analysis showed that ISG20L2 was overexpression significantly in siNC group than in si-ISG20L2 group (Fig.6H).

\section{Discussion}

Pancreatic cancer (PC) is one of the most lethal solid malignancies, remains the increasing incidence in the past decade ${ }^{10}$. Although surgery, chemoradiation and chemotherapy have been improved, overall survival of PC patients remains still poorly. Type 2 diabetes mellitus was associated with overall survival of pancreatic cancer resection and adjuvant chemotherapy ${ }^{11}$. Increasing evidences have shown that patients with PC had concurrent T2DM ${ }^{12}$. Glucose metabolism is associated with T2DM and PDAC. However, the mechanism of glucose metabolism in pancreatic cancer remains unclear.

In the present study, we found that a total of 64 DEGs were identified in GSE20966 of T2DM and a total of 296 DEGs were identified in GSE16515 of pancreatic cancer, respectively. The result showed that $I S G 20 L 2$ was identified as only one common gene in GSE20966 and GSE16515.

We investigated the biological role of ISG20L2 in pancreatic ductal adenocarcinoma (PDAC). Then we found that ISG20L2 was highly expressed in PDAC tumor samples compared with normal tissues, and its expression was associated with a poor prognosis. Functionally, we showed that ISG20L2 could promote cell growth, proliferation, and migration of pancreatic cancer cell lines. 
171 ISG20L2, interferon stimulated exonuclease gene 20kDa-like 2, is a nucleolar 3' to 5' 172 exoribonuclease, a member of a family of vertebrate nucleolar exonucleases.

173 Biochemical evidence demonstrates here that ISG20L2 is also an exoribonuclease that 174 processes RNAs from their 3'-end to their 5'end ${ }^{13}$.

175 To explore the molecular function of ISG20L2, we performed GSEA enrichment 176 analysis. In this study, we observed that hub genes in ISG20L2-high group were 177 statistically significant enriched in pancreatic cancer and metabolism pathways. This 178 revealed that $I S G 20 L 2$ played an important role in pancreatic cancer pathogenesis and 179 glucose metabolism.

180 Immune cell infiltration was verified to be associated with pancreatic cancer ${ }^{14}$. In this 181 work, we found that CD8+ T cells and macrophages were significantly associated with ISG20L2 expression in pancreatic cancer. But the mechanism of ISG20L2 affecting immune cell infiltration in pancreatic cancer is still unknown. This needs us to do further research. ISG20L2 may play a pivotal role in T2DM and pancreatic cancer as the only coexpressed gene. This prompted our interest to explore the relationship. According to above results, we predicted that ISG20L2 was associated with the key enzyme of the glycolysis pathway in pancreatic cancer, for example, GLUT1, PKM, HK2 and LDHA. GLUT1 (glucose transporter 1) is a major glucose transporter, one of the 14 members of the mammalian glucose transporter family, and almost all cellular glucose uptake is regulated by GLUTs ${ }^{15}$. GLUT1 facilitates pancreatic cancer growth and metastasis by mediating glucose transport ${ }^{16-18}$. Pyruvate kinase M (PKM1 and PKM2), a key enzyme in the glycolytic pathway ${ }^{19}$. Previous studies showed that $P K M$ has also played a critical role in glucose metabolism in cancer ${ }^{20-22}$. Hexokinases (HKs), which convert glucose to glucose-6-phosphate, are the key enzymes that regulate glycolysis. HKs include $H K 1, H K 2, H K 3$, and $H K 4$, which are expressed in different tissues. Hexokinase $2(H K 2)$, as the key enzyme regulating the first-step reaction of glycolysis, is overexpressed in many kinds of tumors ${ }^{23}$. HK2 
regulates tumor cellular glucose metabolism to support cell proliferation, migration, and apoptosis resistance, which is required for tumor initiation and development ${ }^{24}$. $L D H$ is a homo- or hetero-tetrameric enzyme composed of two subunits, $\mathrm{M}$ and $\mathrm{H}$, encoded by two highly related genes, $L D H-A$ and $L D H-B^{25}$. Some studies have shown that $L D H A$ regulated glycolysis in progression of pancreatic cancer ${ }^{26,27}$.

Firstly, we analyzed the relationship of the expression of ISG20L2 with the key enzyme of the glycolysis using GEPIA database and found that the expression of ISG20L2 was positively associated with the expression of GLUT1, PKM, HK2 and $L D H A$. These data supported the potential role of ISG20L2 in regulating glucose metabolism in pancreatic cancer. Secondly, above results were validated in vitro. In the current study, we demonstrated that knockdown of ISG20L2 increased GLUT1, PKM, HK2 and LDHA protein levels in pancreatic cancer cells. This relationship was verified in vivo.

\section{Conclusions}

Collectively, we demonstrated that ISG20L2 played a notable role in the process of glycose metabolism, especially in glycolysis. This study provides ISG20L2 as a novel therapeutic target and prognostic biomarker in pancreatic cancer.

\section{Materials and Methods}

\section{Patients and tissue specimens}

Forty PDAC tissues were collected from Tianjin Medical University General Hospital. PDAC tumors were identified by two clinical pathologists. All patients without any preoperative radiotherapy or chemotherapy before surgery were enrolled. All participants accepted written consents in this study. The study was performed under the supervision of the Ethic Committee of Tianjin Medical University General Hospital.

\section{Data downloaded}

GSE20966 and GSE16515 datasets were downloaded from Gene Expression Omnibus (GEO) database (http://www.ncbi.nlm.nih.gov/geo/) ${ }^{28}$. GSE20966 dataset, including 10 pancreatic specimens of type 2 diabetes (T2DM) patients with non-diabetic condition of beta-cells and 10 diabetic condition of beta-cells patients was used to identify T2DM-differentially expressed genes (T2DM-DEGs). Gene-expression 
datasets related to pancreatic cancer GSE16515 dataset with 36 tumor samples and 16 normal samples from GEO database were analyzed to identify PC-differentially expressed genes (PC-DEGs). Pancreatic cancer dataset can be downloaded from the TCGA website (https ://porta l.gdc.cance r.gov/).

\section{Differentially expressed genes (DEGs) identification}

Differential expressed genes were identified between two groups using limma package of R language ${ }^{29}$. Gene expression values of the $|\log 2 \mathrm{FC}|>1$ and adjusted $\mathrm{p}<0.05$ were used to select T2DM-DEGs. However, the $|\log 2 \mathrm{FC}|>2$ and adjusted $\mathrm{p}<0.05$ were used to identify PC-DEGs. Common overlapped DEGs between T2DM-DEGs $\begin{array}{lllll}\text { and } & \text { PC-DEGs were defined by online database VENNY } 2.1\end{array}$ (https://bioinfogp.cnb.csic.es/tools/venny/).

\section{Functional enrichment analysis}

Gene Ontology (GO) and Kyoto Encyclopedia of Genes and Genomes (KEGG) were performed to analyze the molecular functions of T2DM- and PC-DEGs. P $<0.05$ was defined to be significantly enriched. In this study, we presented different biofunctions of DEGs in biological processes (BP), molecular functions (MF), and cellular components (CC), respectively. In addition, GSEA software was used to analyze the molecular function of ISG20L2 expression.

\section{Co-expression of $I S G 20 L 2$ and prognosis prediction}

GEPIA (Gene Expression Profiling Interactive Analysis) is a web-based tool to deliver fast and customizable functionalities based on TCGA and GTEx data. Here we used the Gene Expression Profiling Interactive Analysis (GEPIA) to explore co-expression of ISG20L2 associated with glycolysis and predict prognosis. The key enzymes of glycolysis include GLUT1, HK2, LDHA, PKM.

\section{TIMER}

TIMER (https://cistrome.shinyapps.io/timer/) is a reliable, intuitive tool that provides systematic evaluations of different immune cells infiltration and their clinical impact. In our study, "Gene module" was used to evaluate the correlation between ISG20L2 level and the infiltration of immune cells. 


\section{qRT-PCR}

Total RNA was isolated by using TRIzol reagent (Invitrogen, America) and transformed into cDNA by Reverse Transcription Kit (Takara, Japan). RNA expression was measured by using the SYBR Premix Ex Taq (Takara, Japan). All reactions were repeated at least three times. Each sample was relatively quantified and normalized with GAPDH expression for control. The primer sequences were shown as following:

ISG20L2: F-5' - CTCCTGCACAAGAGCATCCA -3'

R-5'- CGTTGCCCTCGCATCTTC -3'

GAPDH: F-5'- GGTGGTCTCCTCTGACTTCAACAG -3'

\section{R-5'- GTTGCTGTAGCCAAATTCGTTGT -3'}

\section{Cell lines and cultures}

Human normal pancreatic cells (HPDE6-C7) and PDAC cell lines (SW1990) were purchased from Tianjin Createch Company (Tianjin, China) and cultured with Dulbecco's modified Eagle's medium supplemented with $10 \%$ fetal bovine serum (GIBCO, USA). All cells were cultured in an incubator (Thermo Forma, USA) with a humidified atmosphere of $5 \% \mathrm{CO} 2$ at $37^{\circ} \mathrm{C}$.

\section{Si-RNAs transfection}

Gene-specific and negative control siRNAs were synthesized by Createch Biology (Tianjin, China) and transfected into HPDE6-C7 and SW1990 cells for $48 \mathrm{~h}$ using Lipofectamine 2000 ((Invitrogen, USA) according to the manufacturer's protocol.

\section{Si-RNA sequences:}

F-5'-AAUAGAGACACAAAUCCAGGC-3'

\section{R-5'-CUGGAUUUGUGUCUCUAUUGG-3'}

\section{Western blotting assay}

Cells and tissues were lysed in ice-cold RIPA buffer with $1 \mathrm{mM}$ PMSF. Total protein was separated by SDS-PAGE, transferred to PVDF membranes (Millipore, Bed-ford, MA, USA) and blocked with $1 \times$ Blotto in TBST. Primary antibodies used were GLUT1 Antibody (ab115730, 1:1000 dilution; Abcam), HK2 Antibody (ab209847, 1:2000 dilution; Abcam), LDHA Antibody (ab52488, 1:500 dilution; Abcam) PKM1 Antibody 
(ab137791, 1:1000 dilution; Abcam), PKM2 Antibody (ab85555, 1:1000 dilution; Abcam).

\section{Animal experiments}

All animal experiments were approved were approved by the Animal Care Committee of Tianjin Medical University General Hospital. According to the previous study, male or female mice were used for the xenograft subcutaneous implantation model ${ }^{30}$.In this study, 4-week-old female BALB/c nude mice were used and randomly divided into two groups. $5 \times 10^{6} \mathrm{SW} 1990$ cells were subcutaneously injected into female mice. The tumor volumes were measured every 3 days. 4 weeks later, the nude mice were sacrificed and the tumor weights were recorded.

\section{Immunohistochemistry}

IHC was performed on 40 matched formalin fixation and paraffin embedding PDAC tissues. All histologic slides were assessed by two pathologists independently. The degree of positivity was initially classified by scoring both the proportion of positively stained tumor cells and the staining intensities as previously described. The H-score was independently assessed by two professional pathologists.

\section{Statistical analysis}

All statistical analyses were performed by using GraphPad Prism 8 (GraphPad, USA) and R software (version 3.5.2). Comparisons between two groups were analyzed by independent $t$ test. Univariate and multivariate Cox regression analyses were performed to assess the relationship among clinicopathological factors, ISG20L2 expression profiles and prognosis. Overall survival was evaluated with the Kaplan-Meier method, and the log-rank test was employed to evaluate the difference. $\mathrm{P}<0.05$ was considered statistically significant.

\section{Acknowledgements}

Not applicable

\section{Author Contributions}

Jianming Wei and Xibo Gao analyzed genes expression array from the GEO and TCGA database regarding T2DM and PC. Bingbing Ren revised the manuscript. Tong Liu and 
316 Daqing Sun professors supervised this manuscript. All authors read and approved the 317 final manuscript.

\section{Conflict of Interest}

319 The authors declare that they have no competing interests.

320 Funding

321 This study was supported the Tianjin Health Commission Science and Technology

Personnel Cultivation Project (KJ20103).

\section{Declarations}

\section{Ethical Approval and Consent to participate}

325 Not applicable.

\section{Consent for publication}

327 Written informed consent was obtained from the patient for publication of this case report and any accompanying images. A copy of the written consent is available for review by the Editor-in-Chief of this journal.

\section{Availability of supporting data}

331 Not applicable.

\section{References}

1. Sung H, Ferlay J, Siegel RL, et al. Global cancer statistics 2020: GLOBOCAN estimates of incidence and mortality worldwide for 36 cancers in 185 countries. CA Cancer J Clin. 2021.

3. Rahn S, Zimmermann V, Viol F, et al. Diabetes as risk factor for pancreatic cancer: Batabyal P, Vander Hoorn S, Christophi C, Nikfarjam M. Association of diabetes mellitus and pancreatic adenocarcinoma: a meta-analysis of 88 studies. Ann Surg Oncol. 2014;21(7):2453-2462.

Hyperglycemia promotes epithelial-mesenchymal-transition and stem cell properties in pancreatic ductal epithelial cells. Cancer Lett. 2018;415:129-150.

4. Skytte MJ, Samkani A, Astrup A, et al. Effects of carbohydrate restriction on postprandial glucose metabolism, $\beta$-cell function, gut hormone secretion, and satiety in patients with Type 2 diabetes. American journal of physiology Endocrinology and metabolism. 2021;320(1):E7-e18.

5. Mason IC, Qian J, Adler GK, Scheer F. Impact of circadian disruption on glucose metabolism: implications for type 2 diabetes. Diabetologia. 2020;63(3):462-472.

6. Dey P, Li J, Zhang J, et al. Oncogenic KRAS-Driven Metabolic Reprogramming in Pancreatic Cancer Cells Utilizes Cytokines from the Tumor Microenvironment. Cancer Discov. 2020;10(4):608-625.

7. Marselli L, Thorne J, Dahiya S, et al. Gene expression profiles of Beta-cell enriched tissue 
obtained by laser capture microdissection from subjects with type 2 diabetes. PLOS One. 2010;5(7):e11499.

8. Uhlen M, Fagerberg L, Hallstrom BM, et al. Proteomics. Tissue-based map of the human proteome. Science. 2015;347(6220):1260419.

9. Wei J, Gao X, Qin Y, Liu T, Kang Y. An Iron Metabolism-Related SLC22A17 for the Prognostic Value of Gastric Cancer. Onco Targets Ther. 2020;13:12763-12775.

10. Tavakkoli A, Elmunzer BJ, Waljee AK, et al. Survival analysis among unresectable pancreatic adenocarcinoma patients undergoing endoscopic or percutaneous interventions. Gastrointest Endosc. 2020.

11. Kleeff J, Costello E, Jackson R, et al. The impact of diabetes mellitus on survival following resection and adjuvant chemotherapy for pancreatic cancer. Br J Cancer. 2016;115(7):887894.

12. Andersen DK, Korc M, Petersen GM, et al. Diabetes, Pancreatogenic Diabetes, and Pancreatic Cancer. Diabetes. 2017;66(5):1103-1110.

13. Couté $\mathrm{Y}$, Kindbeiter K, Belin S, et al. ISG20L2, a novel vertebrate nucleolar exoribonuclease involved in ribosome biogenesis. Mo/ Cell Proteomics. 2008;7(3):546-559.

14. Michelakos T, Cai L, Villani $V$, et al. Tumor microenvironment immune response in pancreatic ductal adenocarcinoma patients treated with neoadjuvant therapy. J Nat/ Cancer Inst. 2020.

15. Chen J, Cao L, Li Z, LiY. SIRT1 promotes GLUT1 expression and bladder cancer progression via regulation of glucose uptake. Hum Cell. 2019;32(2):193-201.

16. Nagarajan A, Dogra SK, Sun L, et al. Paraoxonase 2 Facilitates Pancreatic Cancer Growth and Metastasis by Stimulating GLUT1-Mediated Glucose Transport. Mol Cell. 2017;67(4):685-701.e686.

17. Huang $\mathrm{S}, \mathrm{Wu} Z$ Z, Cheng Y, Wei W, Hao L. Insulin-like growth factor 2 mRNA binding protein 2 promotes aerobic glycolysis and cell proliferation in pancreatic ductal adenocarcinoma via stabilizing GLUT1 mRNA. Acta Biochim Biophys Sin (Shanghai). 2019;51(7):743-752.

18. Wu L, Qiu W, Sun J. Down regulation of miR-148a is related to enhanced pancreatic cancer pathogenesis through targeting GLUT1. Int J Clin Exp Pathol. 2018;11(10):49504956.

19. Chiavarina B, Whitaker-Menezes D, Martinez-Outschoorn UE, et al. Pyruvate kinase expression (PKM1 and PKM2) in cancer-associated fibroblasts drives stromal nutrient production and tumor growth. Cancer Biol Ther. 2011;12(12):1101-1113.

20. Dayton TL, Jacks T, Vander Heiden MG. PKM2, cancer metabolism, and the road ahead. \#N/A. 2016;17(12):1721-1730.

21. Zahra K, Dey T, Ashish, Mishra SP, Pandey U. Pyruvate Kinase M2 and Cancer: The Role of PKM2 in Promoting Tumorigenesis. Frontiers in oncology. 2020;10:159.

22. Liu F, Ma F, Wang Y, et al. PKM2 methylation by CARM1 activates aerobic glycolysis to promote tumorigenesis. Nat Cell Biol. 2017;19(11):1358-1370.

23. Fan K, Fan Z, Cheng H, et al. Hexokinase 2 dimerization and interaction with voltagedependent anion channel promoted resistance to cell apoptosis induced by gemcitabine in pancreatic cancer. Cancer Med. 2019;8(13):5903-5915.

24. Patra KC, Wang Q, Bhaskar PT, et al. Hexokinase 2 is required for tumor initiation and maintenance and its systemic deletion is therapeutic in mouse models of cancer. Cancer 
Cell. 2013;24(2):213-228.

25. Zhao D, Zou SW, Liu $\mathrm{Y}$, et al. Lysine-5 acetylation negatively regulates lactate dehydrogenase A and is decreased in pancreatic cancer. Cancer Cell. 2013;23(4):464-476.

26. Shi M, Cui J, Du J, et al. A novel KLF4/LDHA signaling pathway regulates aerobic glycolysis in and progression of pancreatic cancer. Clin Cancer Res. 2014;20(16):4370-4380.

27. Cui J, Shi M, Xie D, et al. FOXM1 promotes the warburg effect and pancreatic cancer progression via transactivation of LDHA expression. Clin Cancer Res. 2014;20(10):25952606.

28. Barrett T, Wilhite SE, Ledoux P, et al. NCBI GEO: archive for functional genomics data sets-update. Nucleic Acids Res. 2013;41(Database issue):D991-995.

29. Wei J, Wang J, Gao X, Qi F. Identification of differentially expressed circRNAs and a novel hsa_circ_0000144 that promote tumor growth in gastric cancer. Cancer Cell Int. 2019;19:268.

30. Jin SZ, Meng XW, Sun X, et al. Hepatocyte growth factor promotes liver regeneration induced by transfusion of bone marrow mononuclear cells in a murine acute liver failure model. J Hepatobiliary Pancreat Sci. 2011;18(3):397-405.

\section{Figure legends}

\section{Fig.1. Study flowchart.}

Fig.2. Differentially expressed genes and enrichment analysis in type 2 diabetes mellitus and pancreatic cancer.

Differentially expressed genes in type 2 diabetes mellitus (T2DM) of heatmap (A) and volcano (C). Heatmap (B) and volcano (D) in pancreatic cancer. Red represents upregulated DEGs, green represents downregulated DEGs. (E), (F) T2DM-and PCrelated GO term enrichment for DEGs, respectively. (G), (H) KEGG pathway of T2DM-and PC-related DEGs.

Fig.3. Identification and validation of ISG20L2 expression associated with prognosis.

(A) ISG20L2 is the only one common gene in DEGs by VENNY 2.1. (B) and (C) showed that ISG20L2 expression in GEO database. (D) Validation of the expression of ISG20L2 in TCGA database. (E) low-expression and (F) overexpression of ISG20L2 in The Human Protein Atlas (HPA) database.

(G) RT-qPCR is used to detect the expression of ISG20L2 in pancreatic cancer cells and normal cells. (H) A significant upregulation of ISG20L2 in PDAC tissues $(\mathrm{n}=40)$ compared with normal tissues $(\mathrm{n}=40)$ is observed. The expression of ISG20L2 is significantly associated with overall survival (I), stage (J), and vascular invasion (K), 
430 (L).

431 Fig.4. Gene set enrichment analysis (GSEA) using TCGA database and correlation 432 with immune cell infiltration in TIMER.

433 (A)-(G) only listed the six most common functional gene sets enriched in PC samples 434 with hub genes expressed of ISG20L2 high-expression. CD8+ T cells (H), CD4+ T cells 435 (I), macrophages (J), neutrophils (K), Dendritic cells (L), B cell (M).

436 Fig.5. ISG20L2 enhances glycolysis in PDAC.

437 (A) GLUT1, (B) PKM, (C) HK2 and (D) $L D H A$ are significantly associated with 438 ISG20L2 expression in GEPIA database. (E)-(H) showed that the levels of GLUT1, $439 P K M, H K 2$ and $L D H A$ were examined in si-ISG20L2 and si-NC SW1990 cells .

440 Fig.6. ISG20L2 promotes tumor growth in pancreatic cancer in vitro and vivo.

441 Transwell assays are conducted to examine the effects of ISG20L2 knockdown on 442 PDAC cell migration (A) and invasion (C). (B) The proliferative ability is assessed in 443 ISG20L2-silenced PDAC cells using MTT assay. (D) Xenograft mouse models were 444 used to evaluate the effects of ISG20L2 on PDAC growth. Nude mice were injected 445 subcutaneously with PDAC cells with ISG20L2 knockdown and si-NC. The xenografts 446 were harvested after 1 month. The volumes (E) and weights (F) of the xenografts were 447 measured at the indicated time points. (G) The expression level of ISG20L2 in the 448 xenografts. (H) Representative ISG20L2 immunostaining of the xenografts. 


\section{Figures}

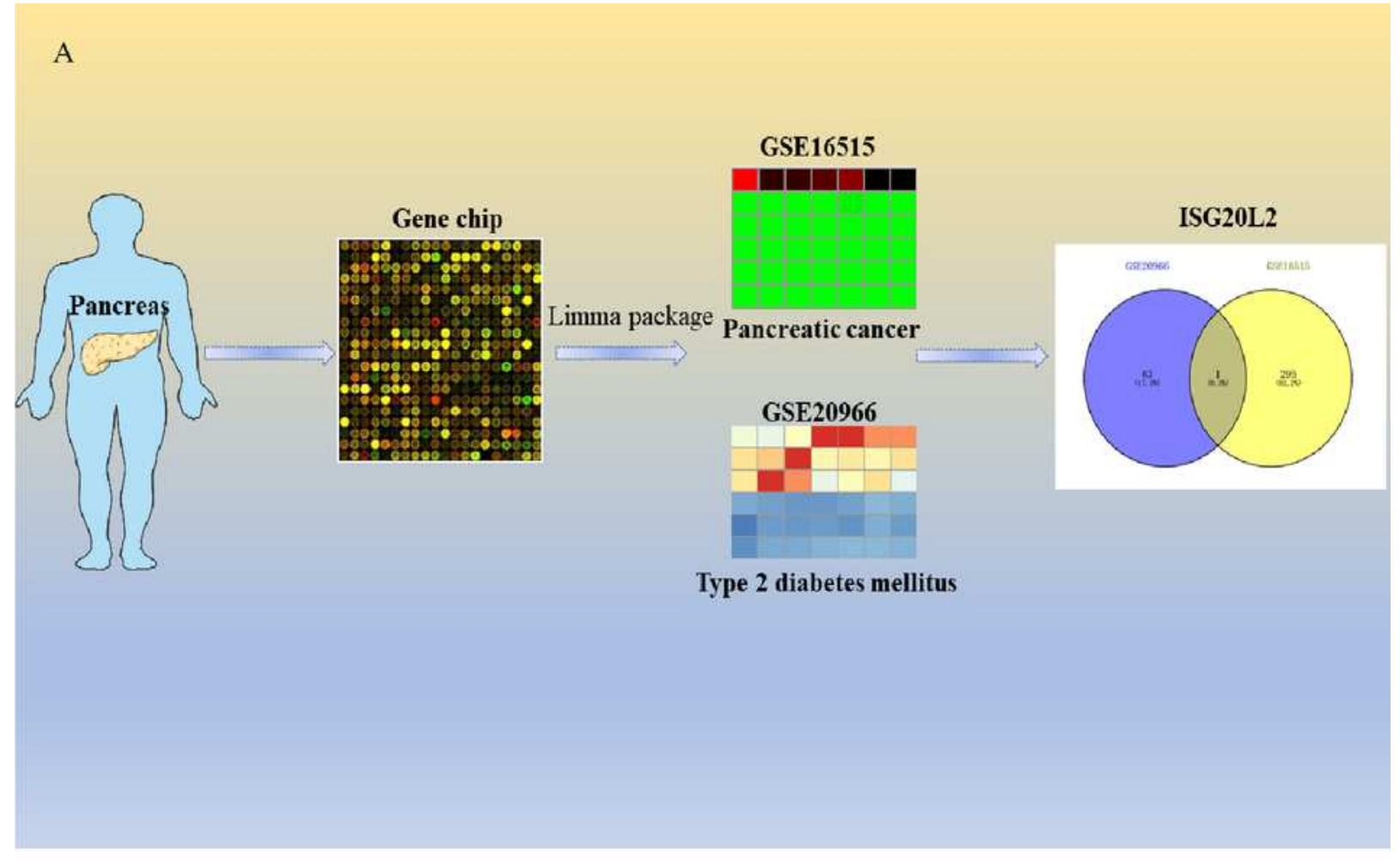

B
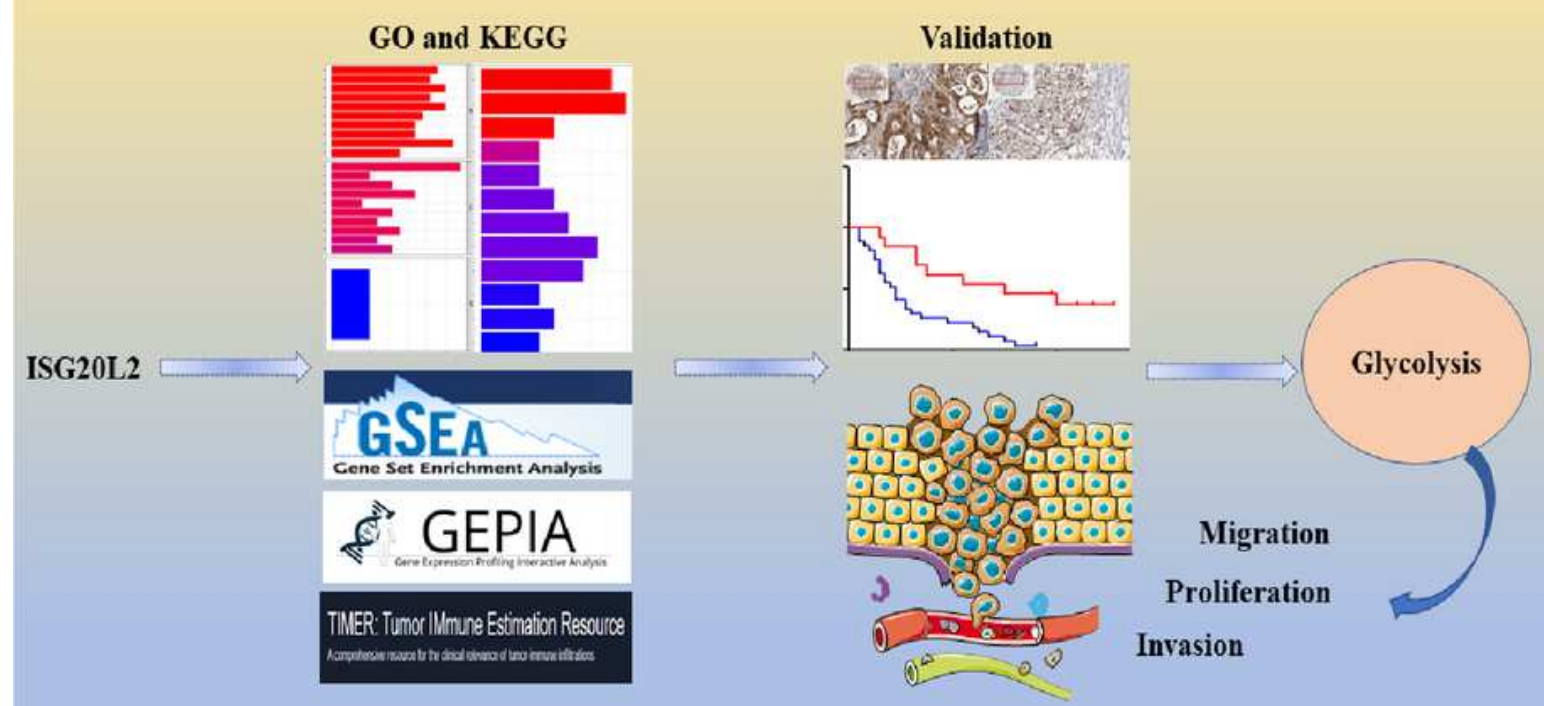

Figure 1

Study flowchart. 
A

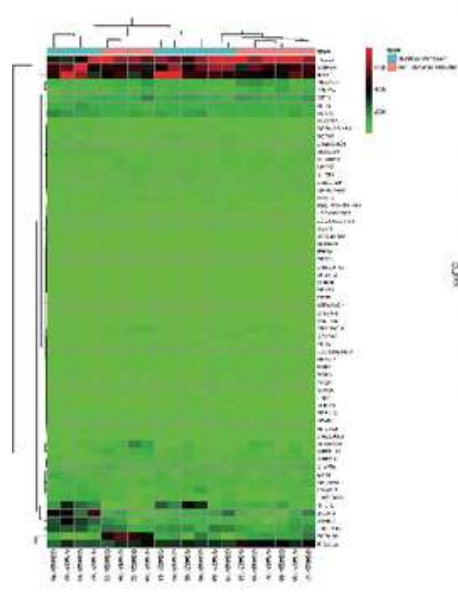

E

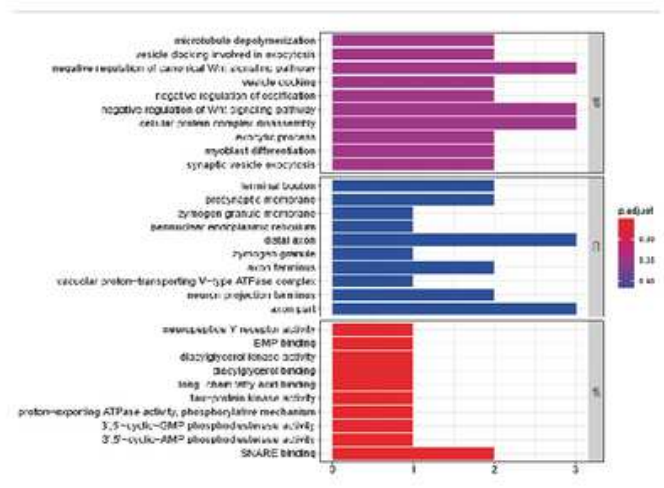

G

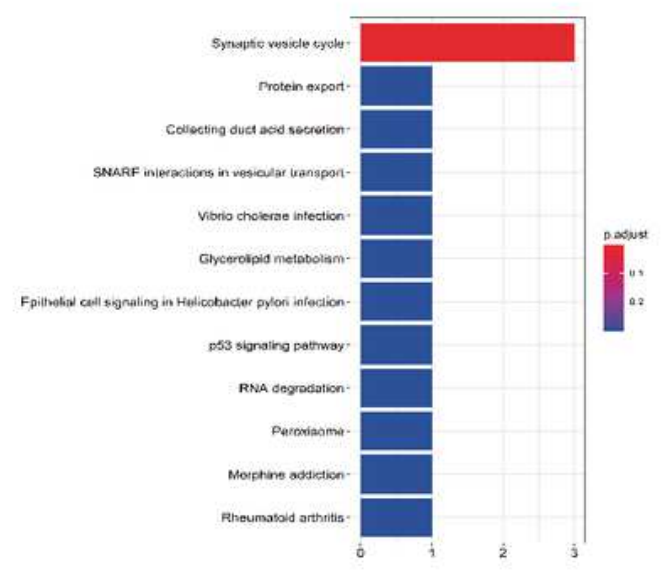

C

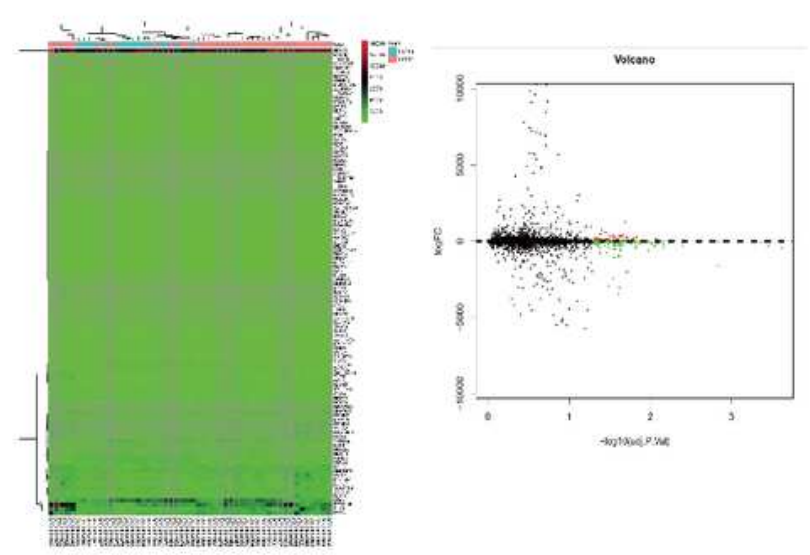

F

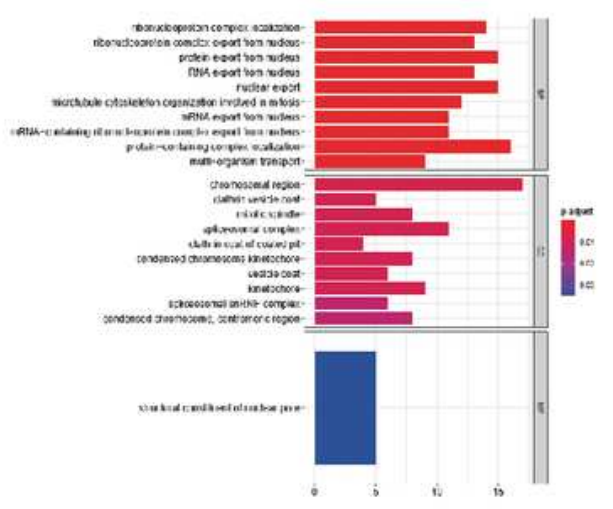

$\mathrm{H}$

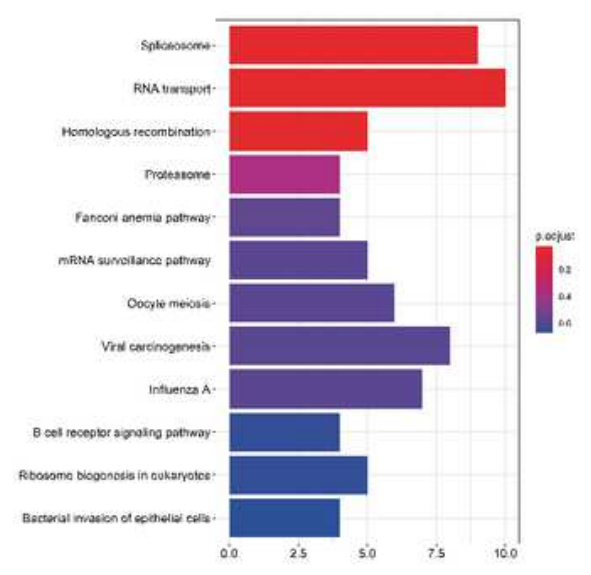

\section{Figure 2}

Differentially expressed genes and enrichment analysis in type 2 diabetes mellitus and pancreatic cancer. Differentially expressed genes in type 2 diabetes mellitus (T2DM) of heatmap (A) and volcano (C). Heatmap (B) and volcano (D) in pancreatic cancer. Red represents upregulated DEGs, green represents downregulated DEGs. (E), (F) T2DM-and PC-related GO term enrichment for DEGs, respectively. (G), (H) KEGG pathway of T2DM-and PC-related DEGs. 
A

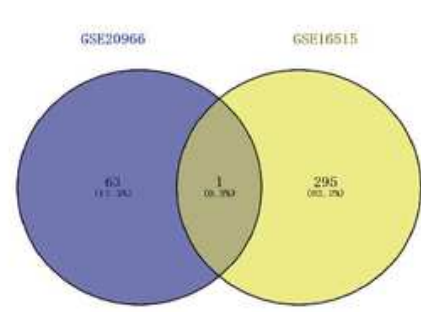

$\mathrm{D}$

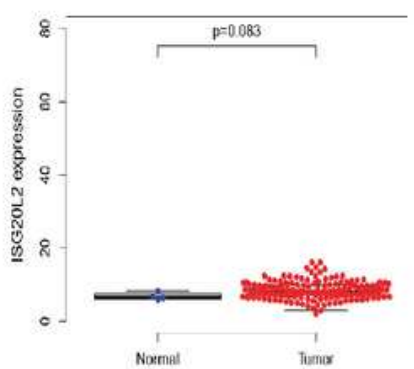

G

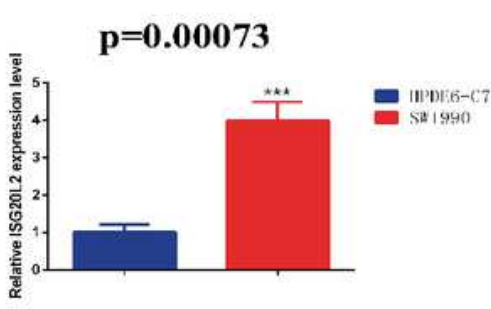

J

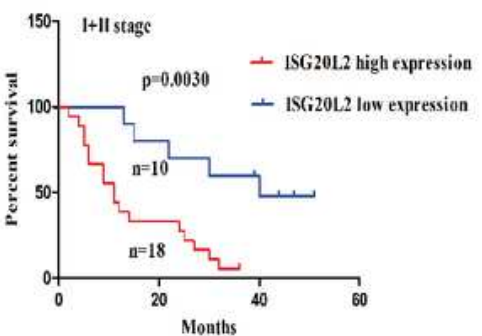

B

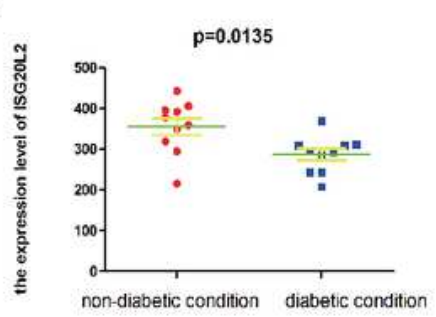

E
C

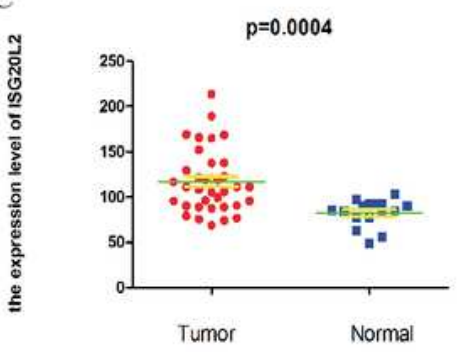

F

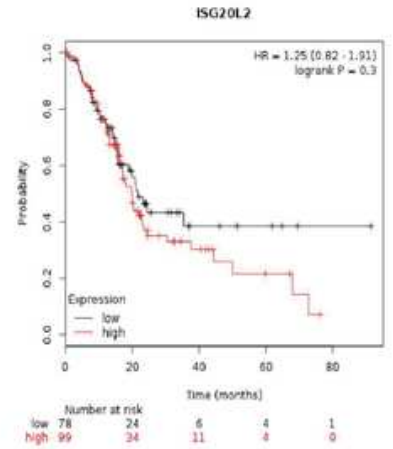

I
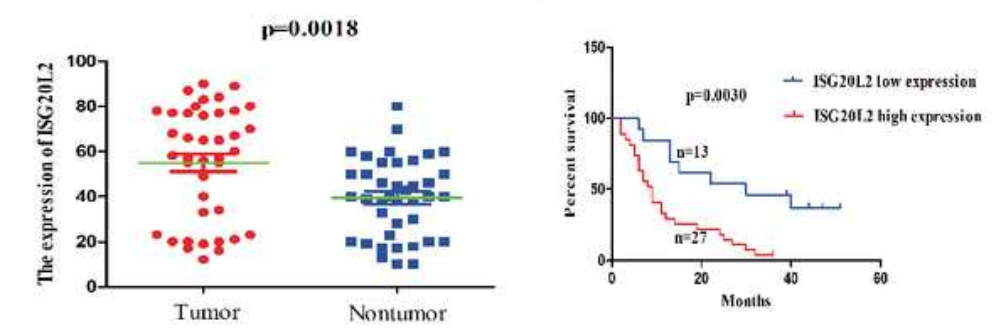

L

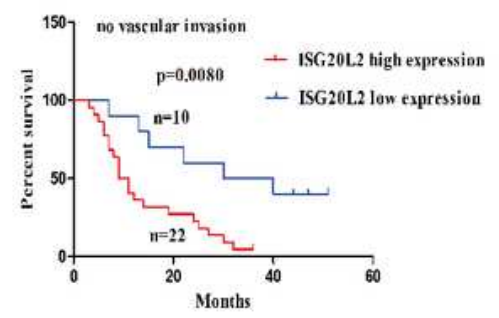

\section{Figure 3}

dentification and validation of ISG20L2 expression associated with prognosis. (A) ISG20L2 is the only one common gene in DEGs by VENNY 2.1. (B) and (C) showed that ISG20L2 expression in GEO database. (D) Validation of the expression of ISG20L2 in TCGA database. (E) low-expression and (F) overexpression of ISG20L2 in The Human Protein Atlas (HPA) database. (G) RT-qPCR is used to detect the expression of ISG20L2 in pancreatic cancer cells and normal cells. (H) A significant upregulation of ISG20L2 in PDAC 
tissues $(n=40)$ compared with normal tissues $(n=40)$ is observed. The expression of ISG20L2 is significantly associated with overall survival $(\mathrm{I})$, stage $(\mathrm{J})$, and vascular invasion $(\mathrm{K}),(\mathrm{L})$.

A

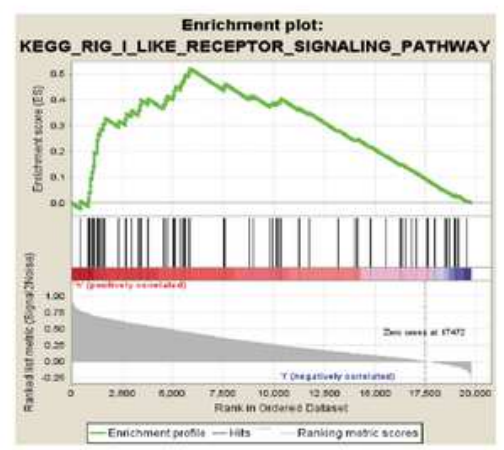

E

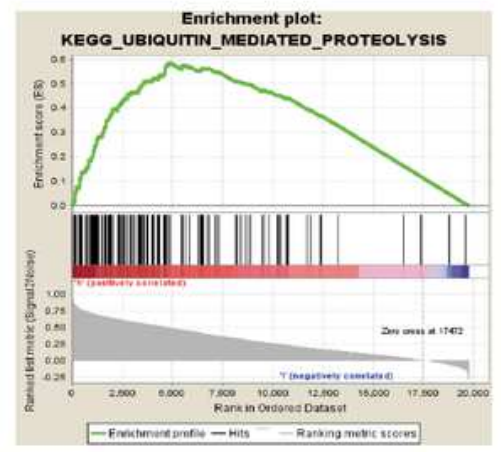

H

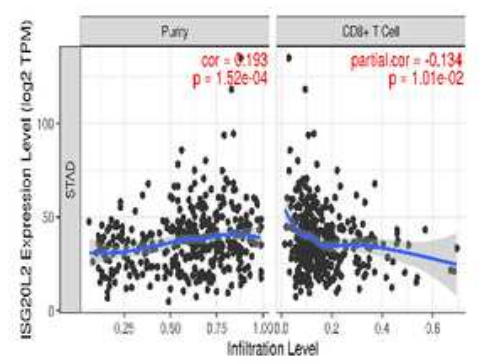

$\mathrm{K}$

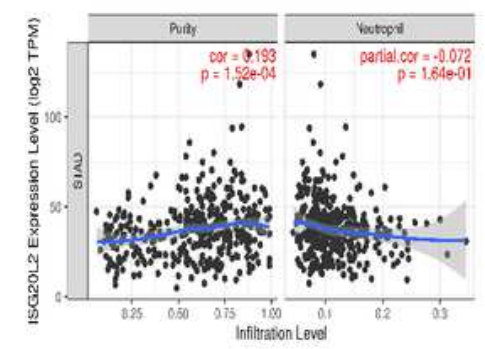

B

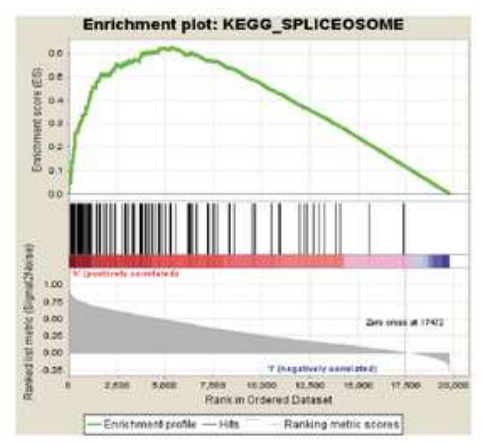

F

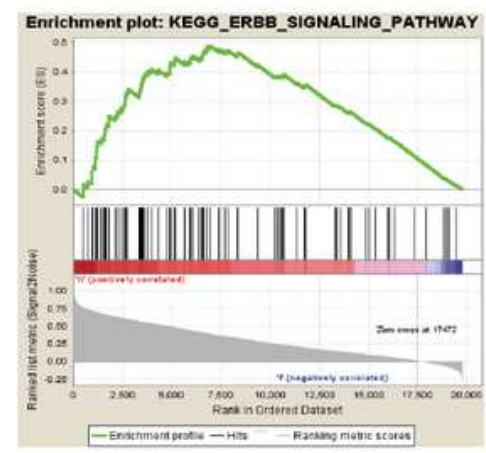

I

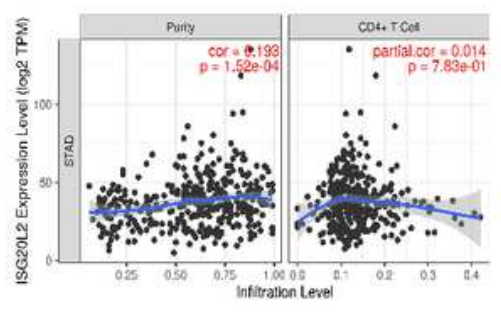

L

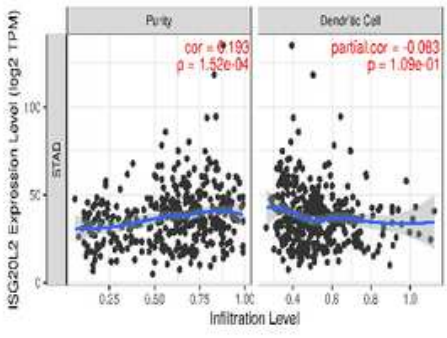

$\mathrm{C}$

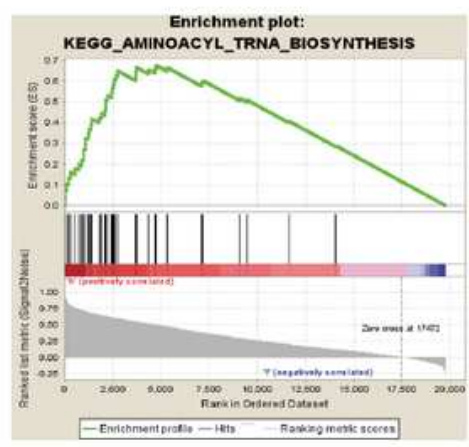

G

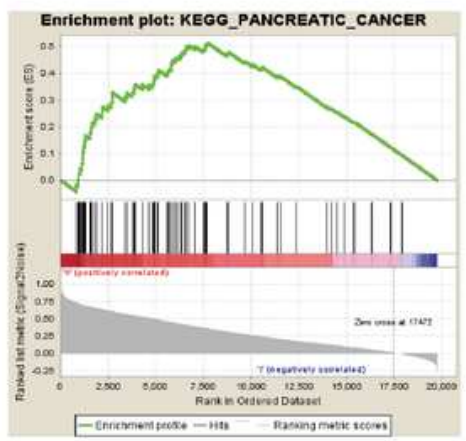

$\mathrm{J}$

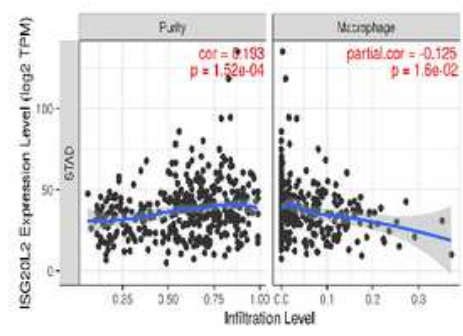

M

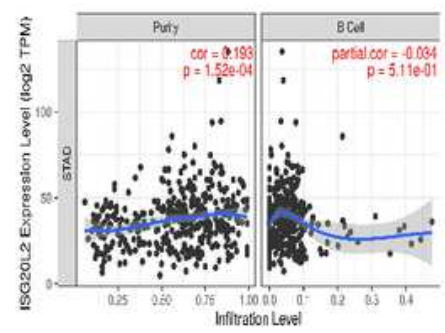

Figure 4

Gene set enrichment analysis (GSEA) using TCGA database and correlation with immune cell infiltration in TIMER. (A)-(G) only listed the six most common functional gene sets enriched in PC samples with hub 
genes expressed of ISG20L2 high-expression. CD8+ T cells (H), CD4+ T cells (I), macrophages (J), neutrophils (K), Dendritic cells (L), B cell (M).

A
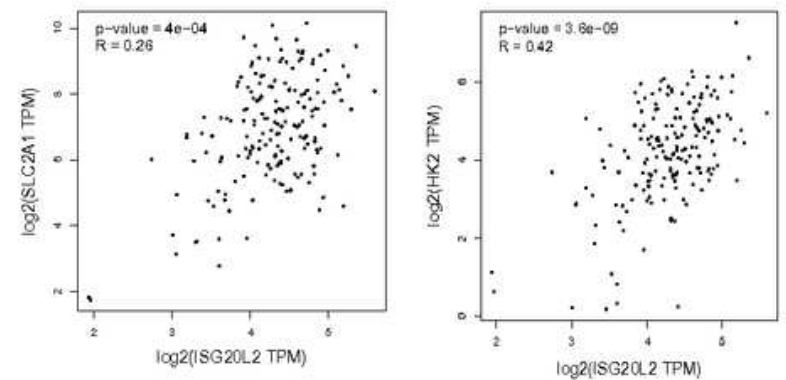

E

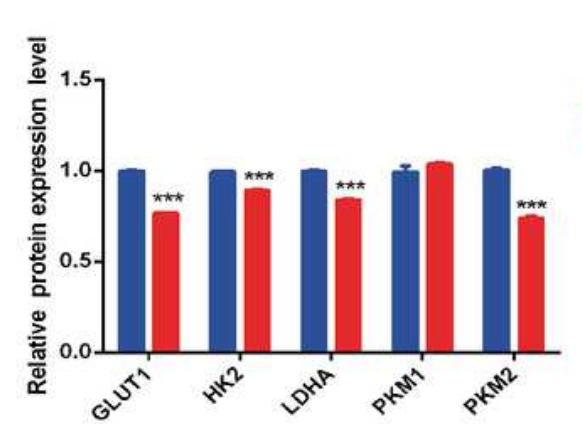

si-NC
si-ISG201.2
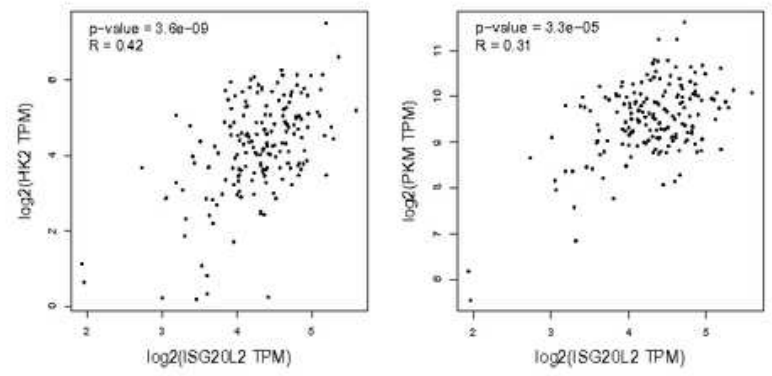

F

si-NC si-ISG20L2

GLUT1

HK2

LDHA

PKM1

PKM2

GAPDH

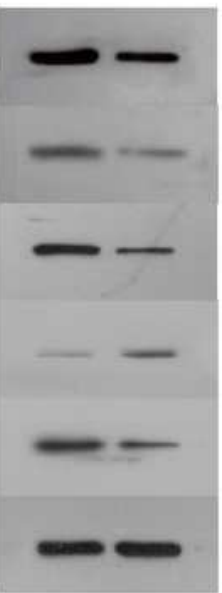

H

G

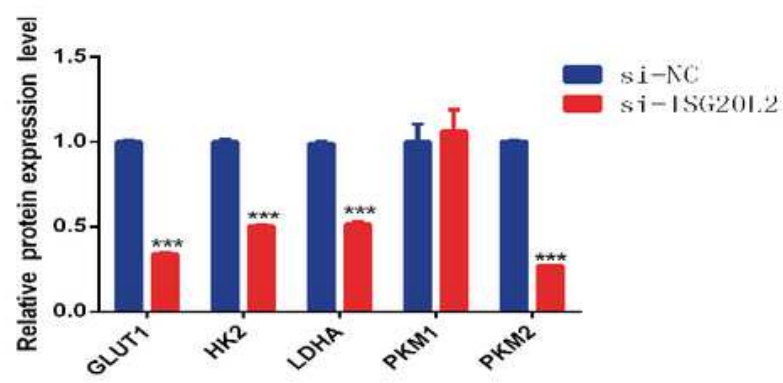

si-NC si-ISG2OL2

GLUT1

HK2

LDHA

PKM 1

PKM2

GAPDH

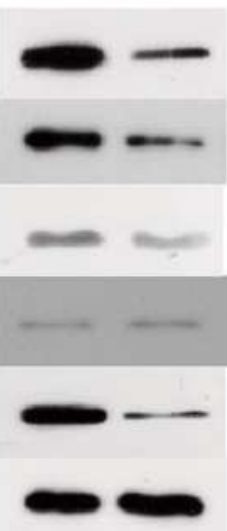

Figure 5

ISG20L2 enhances glycolysis in PDAC. (A) GLUT1, (B) PKM, (C) HK2 and (D) LDHA are significantly associated with ISG20L2 expression in GEPIA database. $(\mathrm{E})-(\mathrm{H})$ showed that the levels of GLUT1, PKM, HK2 and LDHA were examined in si-ISG20L2 and si-NC SW1990 cells. 
A

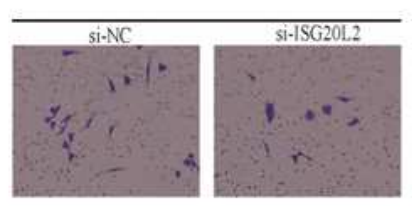

C

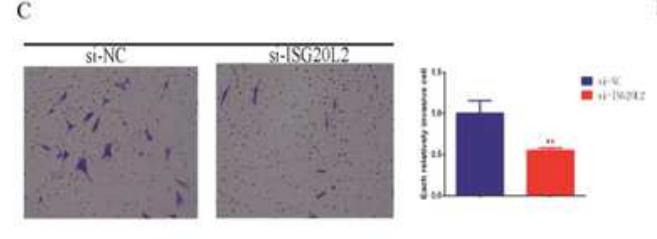

E
B
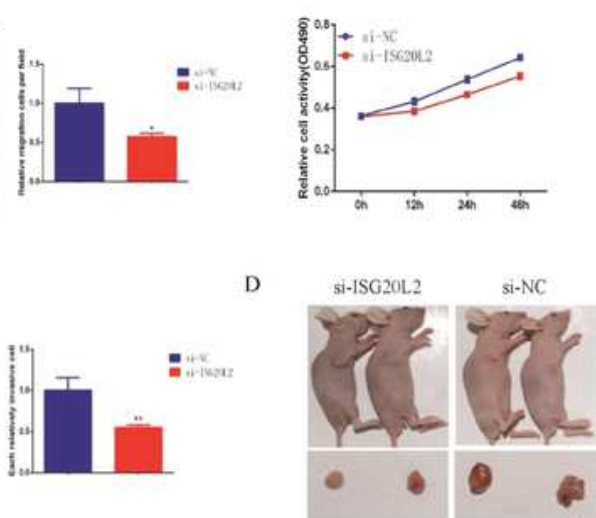

G

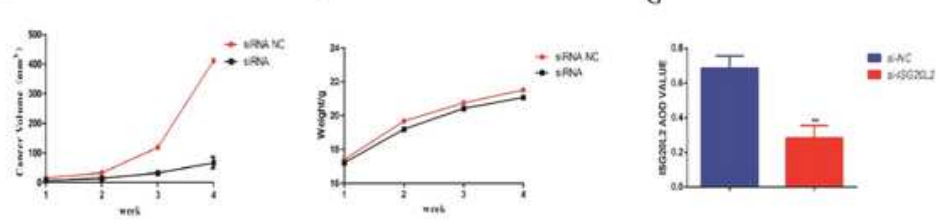

H SW 1990

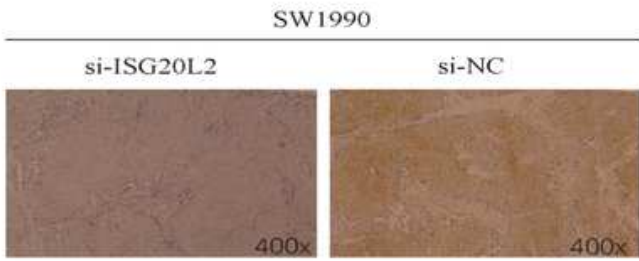

\section{Figure 6}

ISG20L2 promotes tumor growth in pancreatic cancer in vitro and vivo. Transwell assays are conducted to examine the effects of ISG20L2 knockdown on PDAC cell migration (A) and invasion (C). (B) The proliferative ability is assessed in ISG20L2-silenced PDAC cells using MTT assay. (D) Xenograft mouse models were used to evaluate the effects of ISG20L2 on PDAC growth. Nude mice were injected subcutaneously with PDAC cells with ISG20L2 knockdown and si-NC. The xenografts were harvested 
after 1 month. The volumes $(E)$ and weights $(F)$ of the xenografts were measured at the indicated time points. (G) The expression level of ISG20L2 in the xenografts. (H) Representative ISG20L2 immunostaining of the xenografts.

\section{Supplementary Files}

This is a list of supplementary files associated with this preprint. Click to download.

- Table1.pdf 